This is an electronic reprint of the original article. This reprint may differ from the original in pagination and typographic detail.

Author(s): Idini, Andrea; Brown, A.; Langanke, K.; Martínez-Pinedo, G.

Title: $\quad$ Electron Capture Processes in Intermediate Mass stars

Year: $\quad 2014$

Version:

Please cite the original version:

Idini, A., Brown, A., Langanke, K., \& Martínez-Pinedo, G. (2014). Electron Capture Processes in Intermediate Mass stars. In Proceedings NIC XIII : Nuclei in the Cosmos.

Sissa. PoS : Proceedings of Science, NIC XIII, 002.

https://doi.org/10.22323/1.204.0002

All material supplied via JYX is protected by copyright and other intellectual property rights, and duplication or sale of all or part of any of the repository collections is not permitted, except that material may be duplicated by you for your research use or educational purposes in electronic or print form. You must obtain permission for any other use. Electronic or print copies may not be offered, whether for sale or otherwise to anyone who is not an authorised user. 


\section{Electron Capture Processes in Intermediate mass Stars}

\section{A. Idini*}

Institut für Kernphysik, Technische Universität Darmstadt, Schlossgartenstrasse 2, 64289

Darmstadt, Germany

Jyväskylä Yliopisto, Survontie 9, 40500 Jyväskylä, Finland

E-mail: andrea.idini@jyu.fi

\section{A. Brown}

National Superconducting Cyclotron Laboratory, Michigan State University, East Lansing, Michigan 48824, USA

\section{K. Langanke}

Institut für Kernphysik, Technische Universität Darmstadt, Schlossgartenstrasse 2, 64289

Darmstadt, Germany

GSI Helmholtzzentrum für Schwerionenforschung, Planckstraße 1, 64291 Darmstadt, Germany

Frankfurt Institute for Advanced Studies, Ruth Moufang Strasse 1, 60438 Frankfurt, Germany

\section{G. Martínez-Pinedo}

Institut für Kernphysik, Technische Universität Darmstadt, Schlossgartenstrasse 2, 64289

Darmstadt, Germany

GSI Helmholtzzentrum für Schwerionenforschung, Planckstraße 1, 64291 Darmstadt, Germany

Intermediate mass stars develop a degenerate core constituted of $\mathrm{O}, \mathrm{Ne}$ and $\mathrm{Mg}$ during their evolution. As the density in the core increases electron capture sets in igniting $\mathrm{Ne}$ and $\mathrm{O}$ burning. Particularly important is electron capture on ${ }^{20} \mathrm{Ne}$ that has been found recently to be dominated by a second forbidden transition from the $0^{+}$ground state of ${ }^{20} \mathrm{Ne}$ to the $2^{+}$ground state of ${ }^{20} \mathrm{~F}$. We have performed shell-model calculations to determine the transition strength and provide an updated value of the electron capture rate and the expected branching ratio to the corresponding $\beta$-decay process.

XIII Nuclei in the Cosmos,

7-11 July, 2014

Debrecen, Hungary

\footnotetext{
*Speaker.
} 


\section{Introduction}

Electron capture on nuclei is a fundamental process in the in the late stage of stellar evolution $[1,2]$. These processes are particularly crucial in the last phases of intermediate-mass stars $\left(\mathrm{M} \approx 8-10 \mathrm{M}_{\odot}\right)$ where double electron capture on even-even nuclei deplete the $\mathrm{O}-\mathrm{Ne}-\mathrm{Mg}$ core of electrons. This decreases the electron-degeneracy pressure counterbalancing the gravitational collapse, setting the stage for the onset of an electron capture supernova. [3-5]. In other words the Chandrasekar Mass can be considered proportional to the electron abundance $\left(M_{C} \simeq 2.8 \mathrm{M}_{\odot} \times Y_{e}\right)$, hence the processes modifying the $Y_{e}$ of the environment are key to understand the collapse of the star core.

Inside the star, the high density of the environment increases the electron Fermi energy $\left(k_{F} \propto\right.$ $\rho^{1 / 3}$ ) enabling electron captures that are energetically forbidden in vacuum and blocking the phase space of beta decays, reducing the decay rate respect to the vacuum one observed in the lab. In a recent study [6] Martínez-Pinedo and collaborators have evaluated the electron-capture and betadecay rates for several key nuclei for the onset of the electron capture supernova, showing that the forbidden transitions can become dominant over the allowed ones, for relevant density and temperature conditions. However for the case of the forbidden ${ }^{20} \mathrm{Ne}\left(2^{+}, g s\right)+e^{-} \rightarrow{ }^{20} \mathrm{~F}\left(0^{+}, g s\right)+$ $v_{e}$ electron capture, and ${ }^{20} \mathrm{~F}\left(2^{+}, g s\right) \rightarrow{ }^{20} \mathrm{Ne}\left(0^{+}, g s\right)+e^{-}+\bar{v}_{e}$ beta decay, only the experimental upper limit has been used.

We provide here a shell-model calculation of the second-forbidden transition between the two ground states, estimating the Branching Ratio for this decay channel that can eventually be probed experimentally. The strength of the $e$-capture and $\beta$-decay in the astrophysical scenario is also discussed.

\section{Second-Forbidden $\beta$-decay}

Beta-decay and electron capture processes are classified according to the angular momentum unit of the emitted leptons. The case in which the emitted leptons are in $s$-wave state is called "allowed" transition, higher $L$ of the emitted leptons will be referred with higher degree of "forbidness" in the decay. $p$-wave leptons will imply a first-forbidden, $d$-wave a second-forbidden, etc., with consequent reduction of the transition rate. Moreover due to the coupling of the nuclear angular momentum with the spin, at one $\Delta L$ can correspond up to three $\Delta J=L-1, L, L+1$, where case of maximum $\Delta J$ is called "unique" since a single nuclear matrix element is dominant. The selection rule on parity implies $\Delta \pi=(-1)^{\Delta L}$ (cf. [7, 8]).

In a beta decay the energy spectrum of an emitted electron in function of the energy is a continuous function due to the three body nature of the decay, and is given by

$$
N_{i f}^{\beta}(E)=E \sqrt{E-m_{e} c^{2}}\left(E-Q_{i f}\right)^{2} F_{Z}(E) C_{i f}^{\beta}(E),
$$

that is called shape function. In the following we will consider $m_{e} c^{2}=1$ and represent the energies in units of electron mass. $Q_{i f}$ is the nuclear Q-value (in units of $m_{e}$ ) between the final $f$ and initial $i$ states, and $F(E)$ is the Fermi function taking into account the electromagnetic interaction between the electrons and the nucleus. $C_{i f}^{\beta}(E)$ is the shape factor, that is given by the reduced transition 
probability for the nuclear transition,

$$
C_{i f}^{\beta}(E)=g_{A}^{2} \frac{\left|\left\langle f\left|H^{\beta}\right| i\right\rangle\right|^{2}}{2 J_{i}+1}
$$

where $g_{A}$ is the weak axial coupling constant, $H^{\beta}$ is the transition hamiltonian (e.g. for Gamow Teller $\left.H^{\beta}=\sigma \cdot \tau\right) . C_{i f}^{\beta}(E)$ is constant respect to energy for allowed decays, and has a polynomial energy dependence of increasing order for higher forbidness of the decay.

The Decay Rate of the transition is then given by

$$
\lambda^{\beta}=\frac{\ln 2}{K} \int_{1}^{Q} N^{\beta}(E) d E
$$

where $K$ is a constant that can be determined from superallowed Fermi transitions $K=6144 \pm 2 \mathrm{~s}$ [9].

${ }^{20} \mathrm{~F} \beta$-decay to ${ }^{20} \mathrm{Ne}$ has an half life of $11.07 \mathrm{~s}$. The decay is dominated by the allowed transition from the $2^{+}$ground state of ${ }^{20} \mathrm{~F}$ to the $2^{+}$excited state at $1.634 \mathrm{MeV}$ excitation energy of ${ }^{20} \mathrm{Ne}$ [10]; a non-unique first-forbidden decay branch to the $2^{-}$excited state at $4.967 \mathrm{MeV}$ excitation energy of ${ }^{20} \mathrm{Ne}$ has been also observed, with $9 \times 10^{-4}$ Branching Ratio [11]. The decay ${ }^{20} \mathrm{~F}\left(2^{+}, g s\right) \rightarrow{ }^{20} \mathrm{Ne}\left(0^{+}, g s\right)$, which is non-unique second forbidden, is yet to be measured and only an upper limit to the Branching Ratio $=10^{-5}$ is experimentally known [12].

While experimental efforts are undergoing to measure this transition here we give an estimate based on shell model wavefunctions for $s d$-shell nuclei described in [13]. The USDB Hamiltonian [14] is diagonalized in the $1 s$ and $0 d$ configuration space, considering $0 s$ and $0 p$ shells fully occupied.

Then, making use of the transition density expressed in the harmonic oscillator basis, we use the description of $[15,16]$ of the forbidden transition matrix elements in terms of nuclear matrix elements in order to calculate the shape factor and then the decay rate making use of Eq. (2.3).

The resulting electron energy spectrum $N_{g s, g s}^{\beta}(E)$ is shown in Fig. 1, and the evaluated total Branching Ratio is $1.3 \times 10^{-6}$. However the allowed $2^{+} \rightarrow 2^{+}$transition will be dominant up to the maximum emitted electron Energy at $5.901 \mathrm{MeV}$. The experimental effort to measure this transition will eventually have to focus on the sector between 5.9 and $7.5 \mathrm{MeV}$ that contains $\approx 10 \%$ of the total forbidden decay's emission strength.

\section{Electron capture in intermediate mass stars}

In recent simulations [4] has been pointed out that the typical range of temperature and densities for the onset of electron-capture supernova are $T \approx 10^{8}-10^{10} \mathrm{~K}\left(\approx 10^{3}-10^{5} \mathrm{eV}\right), \rho \approx$ $10^{9}-10^{10} \mathrm{~g} / \mathrm{cm}^{3}$ that imply an electron chemical potential $\mu_{e} \approx 5-11 \mathrm{MeV}$.

Following the formalism in [6], at finite temperature there is a probability $e^{-E_{i} / k T}$ of thermally exciting a state $i$. There is then a competition between temperature, so the excitation of the ${ }^{20} \mathrm{Ne}\left(2^{+}, 1.64 \mathrm{MeV}\right)$ state thus enabling the allowed transition to the ${ }^{20} \mathrm{~F}\left(2^{+}, g s\right)$, and the slow decay rate of the forbidden transition directly from the ${ }^{20} \mathrm{Ne}\left(0^{+}, g s\right)$. At high temperature the probability of thermal excitation will take over the one of the forbidden ground-state to ground-state transi- 


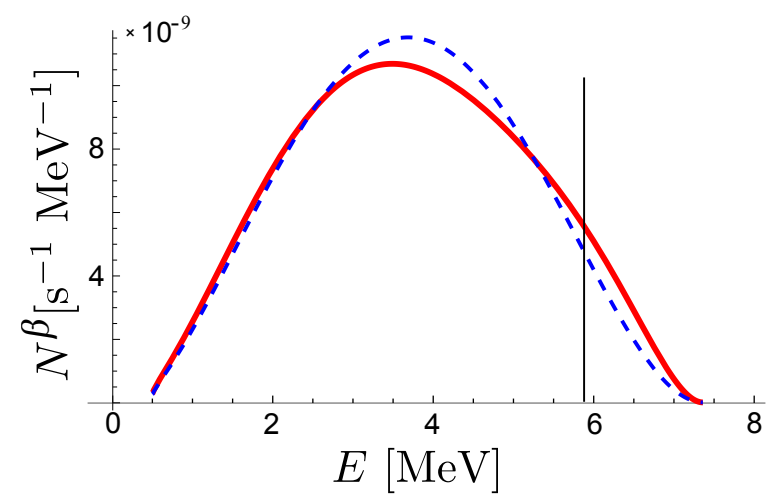

Figure 1: Electron emission shape function in function of the energy, considering the shape factor $C_{g s, g s}^{\beta}(E)$ as a constant (blue dashed line) or with the full energy dependence (red solid line). The black vertical line delimitates the maximum emitted electron Energy of the allowed decay at 5.9 MeV. The dependence to the energy of the shape factor has consequence especially on the high-Energy part of the emission. Considering only the average $\left\langle C_{g s, g s}^{\beta}\right\rangle$ and not the full energy dependence, results in only $7 \%$ of the emission strength above $Q_{g s, 2^{+}}=5.901 \mathrm{MeV}$.

tion, at low temperature the ground state to ground state transition will be the only available within a certain range for the chemical potential, so until the $1^{+}$excited state of ${ }^{20} \mathrm{~F}$ will be accessible.

Also the decay rate has to consider the phase space blocked or allowed by the electron distribution

$$
\begin{aligned}
\lambda^{\beta} & =\frac{\ln 2}{K} \int_{1}^{Q_{i f}} N^{\beta}(E)\left(1-S_{e}\left(E, T, \mu_{e}\right)\right) d E, \\
\lambda^{e c} & =\frac{\ln 2}{K} \int_{E_{l}}^{\infty} N^{e c}(E) S_{e}\left(E, T, \mu_{e}\right) d E,
\end{aligned}
$$

where $E_{l}$ is the energy threshold, in units of electron mass, which is given by

$$
E_{l}=\left\{\begin{array}{ll}
1 & \text { if } Q_{i f}>-1 \\
\left|Q_{i f}\right| & \text { if } Q_{i f}<-1
\end{array},\right.
$$

and $S_{e}$ is the electron distribution function, that considering the conditions of the electron gas at temperature $T$ and chemical potential $\mu_{e}$, follows the Fermi-Dirac distribution

$$
S_{e}\left(E, T, \mu_{e}\right)=\frac{1}{\exp \left\{\frac{E-\mu_{e}}{k T}\right\}+1} .
$$

The estimated decay rates, in function of the density, are compared with the previous results of [6] in Fig. 2 where can be seen that, while the energy dependence of the shape factor does not play a relevant role in this case, the reduction of almost one order of magnitude respect to the current experimental upper limit imply a noticeable effect. However the forbidden transition remain dominant for a relevant range of densities and temperatures.

From Eqs. (3.1,3.2) we can consider the decay rate in function of density and temperature, and thus verify in which conditions a certain transition will be dominant over the others (cf. Fig. $3,4)$. 


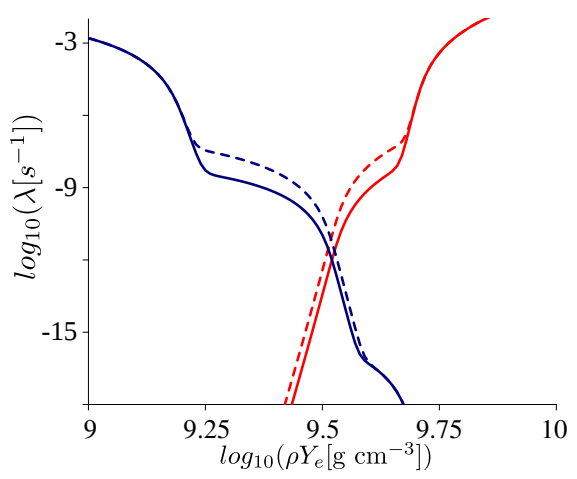

Figure 2: Electron Capture (in red) and beta decay (in blue) rate, at $T=4 \times 10^{8} \mathrm{~K}$. The electron capture decay rate is small at low densities and large at high densities, while for the beta decay rate the opposite is true. In comparison the results making use of the experimental upper limit as energy independent transition matrix element, as in [11] (dashed lines), with the results of the present study (solid lines).
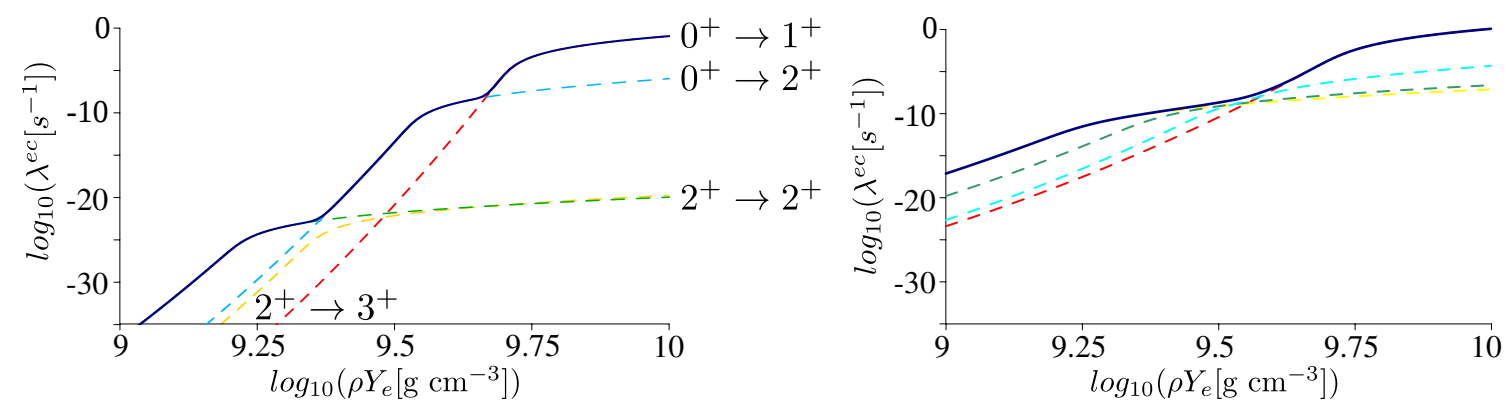

Figure 3: Electron Capture decay rate for ${ }^{20} \mathrm{Ne}\left(0^{+}\right) \rightarrow{ }^{20} \mathrm{~F}\left(1^{+}\right)$(dashed red line), ${ }^{20} \mathrm{Ne}\left(0^{+}\right) \rightarrow{ }^{20} \mathrm{~F}\left(2^{+}\right)$ (dashed light blue line), ${ }^{20} \mathrm{Ne}\left(2^{+}\right) \rightarrow{ }^{20} \mathrm{~F}\left(2^{+}\right)$(dashed green line), ${ }^{20} \mathrm{Ne}\left(2^{+}\right) \rightarrow{ }^{20} \mathrm{~F}\left(3^{+}\right)$(dashed yellow line) transitions, and the sum (solid blue line), in function of the density, for $T=4 \times 10^{8} \mathrm{~K}$ (left panel) and $T=1 \times 10^{9} \mathrm{~K}$ (right panel).
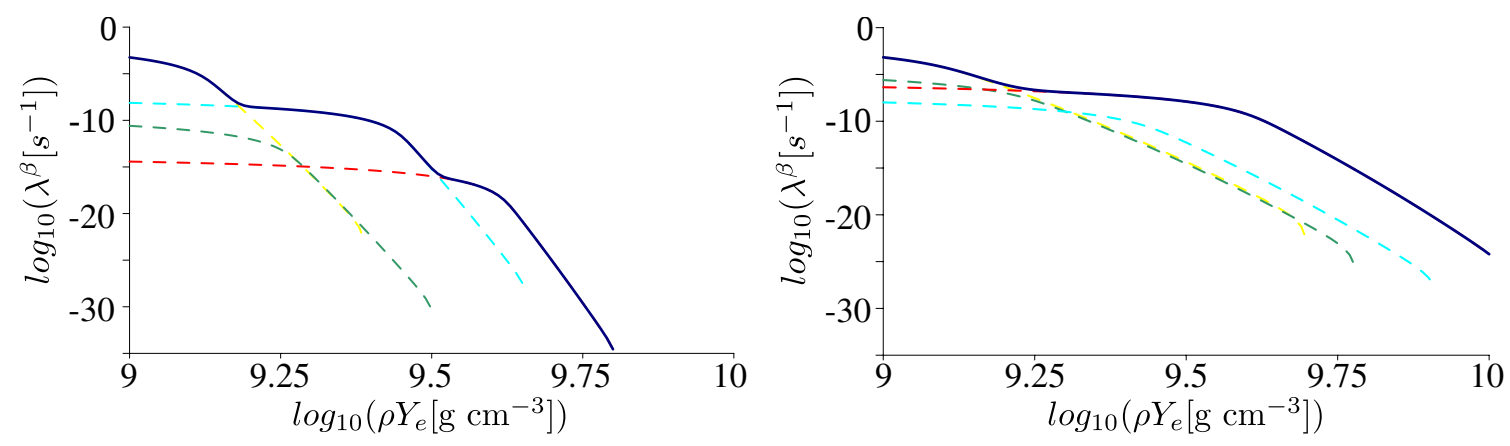

Figure 4: Beta decay rate for ${ }^{20} \mathrm{~F}\left(1^{+}\right) \rightarrow{ }^{20} \mathrm{Ne}\left(0^{+}\right)$(dashed red line), ${ }^{20} \mathrm{~F}\left(2^{+}\right) \rightarrow{ }^{20} \mathrm{Ne}\left(0^{+}\right)$(dashed light blue line), ${ }^{20} \mathrm{~F}\left(2^{+}\right) \rightarrow{ }^{20} \mathrm{Ne}\left(2^{+}\right)$(dashed green line), ${ }^{20} \mathrm{~F}\left(3^{+}\right) \rightarrow{ }^{20} \mathrm{Ne}\left(2^{+}\right)$(dashed yellow line) transitions, and the sum (solid blue line), in function of the density, for $T=4 \times 10^{8} \mathrm{~K}$ (left panel) and $T=1 \times 10^{9} \mathrm{~K}$ (right panel).

\section{Acknowledgments}

This work has been supported by the Helmholtz Association through the Nuclear Astrophysics Virtual Institute (VH-VI-417) and the Helmholtz International Center for FAIR within the frame- 
work of the LOEWE program launched by the state of Hesse

\section{References}

[1] H. A. Bethe, "Supernova mechanisms," Reviews of Modern Physics, vol. 62, pp. 801-866, Oct. 1990.

[2] K. Langanke and G. Martínez-Pinedo, "Nuclear weak-interaction processes in stars," Rev. Mod. Phys., vol. 75, p. 819, 2003.

[3] K. Nomoto, "Evolution of 8-10 solar mass stars toward electron capture supernovae. I - Formation of electron-degenerate O + NE + MG cores," The Astrophysical Journal, vol. 277, pp. 791-805, Feb. 1984.

[4] S. Jones, R. Hirschi, K. Nomoto, T. Fischer, F. X. Timmes, F. Herwig, B. Paxton, H. Toki, T. Suzuki, G. Martínez-Pinedo, Y. H. Lam, and M. G. Bertolli, "Advanced burning stages and fate of 8-10 M stars," The Astrophysical Journal, vol. 772, no. 2, p. 150, 2013.

[5] H. Möller, "Electron capture processes in intermediate mass star," vol. in this volume, 2015.

[6] G. Martínez-Pinedo, Y. H. Lam, K. Langanke, R. G. T. Zegers, and C. Sullivan, "Astrophysical weak-interaction rates for selected A $=20$ and A = 24 nuclei," Phys. Rev. C, vol. 89, p. 045806, 2014.

[7] H. Behrens and W. Bühring, Electron Radial Wave Functions and Nuclear Beta-Decay. The International Series of Monographs on Physics Series, Clarendon Press, 1982.

[8] J. Suhonen, From Nucleons to Nucleus: Concepts of Microscopic Nuclear Theory. Theoretical and Mathematical Physics, Springer, 2007.

[9] J. C. Hardy and I. S. Towner, "Superallowed $0^{+} \rightarrow 0^{+}$nuclear $\beta$ decays: A new survey with precision tests of the conserved vector current hypothesis and the standard model," Phys. Rev. C, vol. 79, p. $055502,2009$.

[10] C. Wong, "Beta decay of ${ }^{20}$ F," Phys. Rev., vol. 95, p. 761, Aug 1954.

[11] D. E. Alburger and E. K. Warburton, "Beta-ray branching in the decay of ${ }^{20}$ F," Phys. Rev. C, vol. 24, p. 296, 1981.

[12] F. Ajzenberg-Selove, "Energy levels of light nuclei A = 18-20," Nucl. Phys. A, vol. 475, p. 1, 1987.

[13] B. H. Wildenthal, M. S. Curtin, and B. A. Brown, "Predicted features of the beta decay of neutron-rich sd-shell nuclei," Phys. Rev. C, vol. 28, p. 1343, 1983.

[14] B. A. Brown and W. A. Richter, "New "usd" hamiltonians for the $s d$ shell," Phys. Rev. C, vol. 74, p. 034315, Sep 2006.

[15] H. Behrens and W. Bühring, "Nuclear beta decay," Nucl. Phys. A, vol. 162, no. 1, p. 111, 1971.

[16] W. Bambynek, H. Behrens, M. H. Chen, B. Crasemann, M. L. Fitzpatrick, K. W. D. Ledingham, H. Genz, M. Mutterer, and R. L. Intemann, "Orbital electron capture by the nucleus (and erratum)," Rev. Mod. Phys., vol. 49, p. 77, 1977. 